\title{
Oперативная память от GSI Technology: рекордные параметры и высокая надежность
}

\author{
Е. Павлюкович ${ }^{1}$
}

УДК 621.3.049.774 | ВАК 05.27.01

\begin{abstract}
Основанная в 1995 году в Купертино (Калифорния, США) fabless-компания GSI Technology специализируется на разработке высокопроизводительной статической оперативной памяти, занимая в этой области ведущие позиции в мире. Она предлагает микросхемы СОЗУ, которые отличаются очень большим объемом и высокой скоростью доступа к произвольной ячейке памяти во всем адресном пространстве, малой задержкой, широкой полосой пропускания и низким энергопотреблением. GSI Technology обеспечивает чрезвычайно длительный период поддержки серийного выпуска своих изделий, короткие сроки поставки и высокий уровень технического сопровождения. До недавнего времени компания не имела локального представительства в СНГ, и по этой причине ее продукция не была известна широкому кругу разработчиков этих стран. Цель данной статьи - восполнить пробел информации о компании и выпускаемых ею устройствах.
\end{abstract}

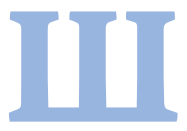

таб-квартира GSI расположена в Саннивейле (США), офисы и представительства размещены по всему миру: в США, Канаде, Израиле, на Тайване. Офис, отвечающий за работу с заказчиками из Европы и России, находится в Израиле. Локальный офис, работающий с компаниями из СНГ, - в Минске. Центр технической поддержки пользователей микросхем расположен в Атланте (Джорджия, США), а вычислителей для машинного зрения - в Израиле.

\section{ГОНКА ЗА ПРОИЗВОДИТЕЛЬНОСТЬЮ}

Небывалый рост объема обрабатываемых и передаваемых данных требует сегодня применения все более высокопроизводительных решений, однако само значение термина "высокая производительность" меняется. Раньше основным параметром статической памяти была задержка чтения (Read Latency) - интервал времени между отправкой адреса и выдачей данных из памяти. При этом задержка записи не принималась в расчет, так как она примерно одинаковая у SRAM и DRAM (DDR2/3/4/5).

Затем производители памяти сфокусировались на повышении тактовой частоты. С увеличением объема информации, которую необходимо сохранять в SRAM, производители сетевого оборудования начали оценивать память по полосе пропускания.

Представитель компании GSI Technology в странах СНГ,

тел.: +7926 102-75-08, y.pauliukovich@semicom.by.
С появлением быстрого Интернета возросло значение времени обработки запроса коммутатора к серверу. Стало очевидным, что полоса пропускания не является критичным параметром. Основная задержка определяется временем ожидания ответа о наличии информации по указанному адресу. Чем быстрее коммутатор опрашивает адреса, тем более производительна система.

Таким образом, для понимания сути термина "высокая производительность" в настоящее время необходимо обращать внимание на оба параметра: полосу пропускания (Data Bandwidth) и скорость доступа (Transaction Rate). Компания GSI Technology, также как и другие производители, стремится в каждом новом семействе микросхем SRAM увеличивать производительность как минимум вдвое (рис. 1).

\section{ПЕРИОД ЖИЗНИ МИКРОСХЕМ}

Компания GSI Technology не снимает с производства каждую новую серию в течение семи лет с момента запуска. На самом деле, период выпуска некоторых микросхем гораздо дольше. С целью уменьшения себестоимости и совершенствования изделий выпускаются новые релизы микросхем, но изготовление предыдущего поколения продолжается еще некоторое время, что дает потребителю возможность адаптивного перехода на новую модель. Например, микросхема GS832, которую начали выпускать в конце 2001 года, претерпела всего одну модернизацию в 2011 году, и обновленная версия GS832A выпускается до 


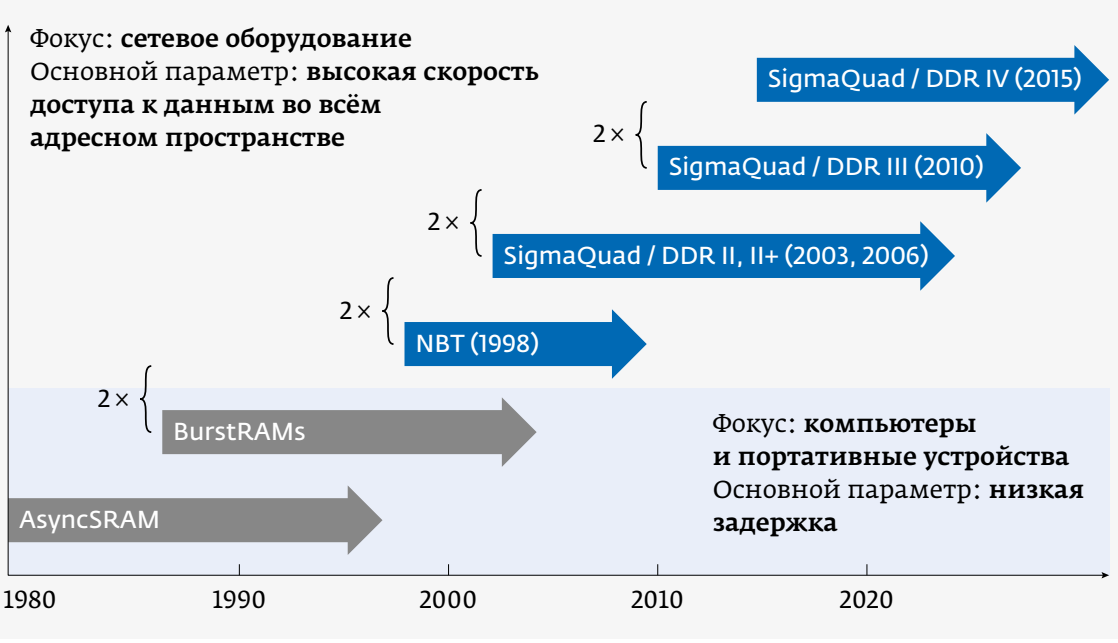

Рис. 1. Эволюция SRAM

настоящего времени. В большинстве случаев оптимальные характеристики микросхемы удается получить уже во втором релизе. GSI стремится сделать все возможное, чтобы обеспечить полную преемственность, сохраняя тип корпуса, расположение выводов и программное обеспечение. При выпуске каждого последующего релиза инкрементируется всего лишь один символ в децимальном номере.

\section{ТЕХНОЛОГИЯ ПРОИЗВОДСТВА}

GSI Technology является fabless-компанией и не обладает собственными фабриками по производству пластин, кристаллов или корпусов. Для производства микросхем компания работает в кооперации с известными тайваньскими фабриками, такими как TSMС и РТС. На территории фабрик работают штатные операционисты GSI Technology, которые координируют производство и осуществляют отправку образцов потенциальным потребителям. Микросхемы проходят полный цикл испытаний в лабораториях KYEC и Global Test. Фундаментальным отличием GSI Technology от других производителей является концепция использования одного мастер-кристалла в нескольких микросхемах. Такая модель обеспечивает важные преимущества, в частности конкурентные цены, короткий срок производства (на складе всегда поддерживается запас из нескольких тысяч мастер-кристаллов), длительный срок жизни микросхемы, универсальность микросхем с программно-управляемыми функциями, значительно меньшее количество изделий в минимальном заказе.

В настоящее время GSI выпускает самую широкую продуктовую линейку SRAM - более 15 тыс. децимальных номеров. Этот факт дает возможность для разработчиков радиоэлектронного оборудования выбрать микросхему по требуемым параметрам и гибко настроить ее под свою задачу.
Микросхемы снимаются с производства только по двум причинам: закрытие фабрики поставщика или отсутствие спроса.

\section{АССОРТИМЕНТ ПРОДУКЦИИ}

На рис. 2 представлены основные семейства микросхем GSI Technology. Несмотря на то, что корпоративная политика GSI Technology заключается в разработке и производстве высокопроизводительной статической памяти большого объема, немалым спросом на рынке также пользуются микросхемы асинхронной SRAM OT GSI. Связано это, прежде всего, с тем, что другие производители прекратили выпуск некоторых своих линеек, и их клиенты вынуждены искать аналоги у более надежного производителя. Микросхемы асинхронной SRAM от различных производителей полностью взаимозаменяемы. Для подбора аналогов на сайте GSI реализован удобный поиск - Cross Reference. Асинхронная память GSI представлена достаточно широкой линейкой продуктов. Предлагаемые варианты микросхем:

- объем памяти от 1 до 9 мбит;

- архитектурах4/8/16/24/32;

- время доступа 8/10/12 нс;

- напряжение питания 3,3 B;

- корпуса TSOP-II, 119-ВGA и FPBGA 6×8 мм или 6×10 мм;

- индустриальное или коммерческое исполнение.

GSI производит микросхемы памяти, которые отличаются не только самой высокой производительностью в мире (скорость доступа до 2 млрд транзакций в секунду, полоса пропускания 163 Гбит/с), но и самой большой информационной емкостью во всех семействах до 288 Мбит. Если в качестве примера взять семейства NBT или SyncBurst, которые широко применяются в среднепроизводительных системах и радиолокации, то ближайшие аналоги от других производителей характеризуются в четыре раза меньшим объемом памяти - до 72 мбит.

В настоящее время только GSI Technology производит SRAM с напряжением питания 1,8 B и сигналами тактирования амплитудой 1,8 В. Это позволяет упростить схемотехническое решение, когда на плате присутствуют только 1,8-В сигналы. Отличить эти микросхемы можно по символу $V$ в конце децимального номера, например GS8161Z36DGD-200IV. Большой объем не всегда означает большой корпус. Микросхемы семейства NBT и SyncBurst объемом 144 и 288 мбит выпускаются в корпусе меньших размеров, чем объемом 72 Мбит (табл. 1). 165-BGA (15×13 мм) - самый компактный корпус у всех производителей. На сегодняшний день только 

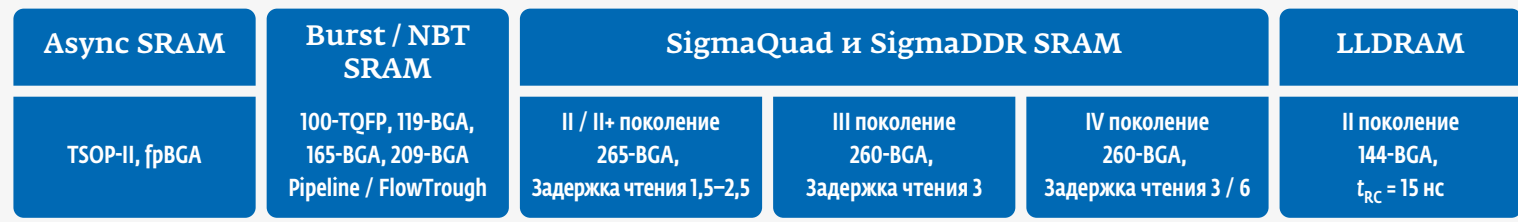

\section{6 Мбнт}
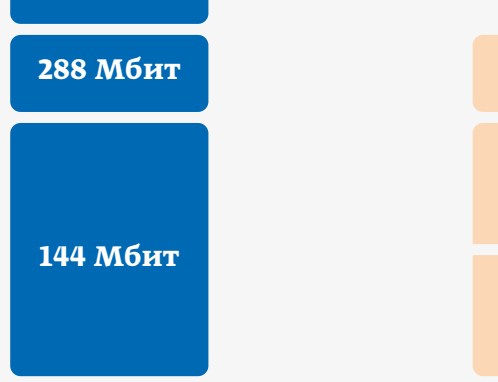

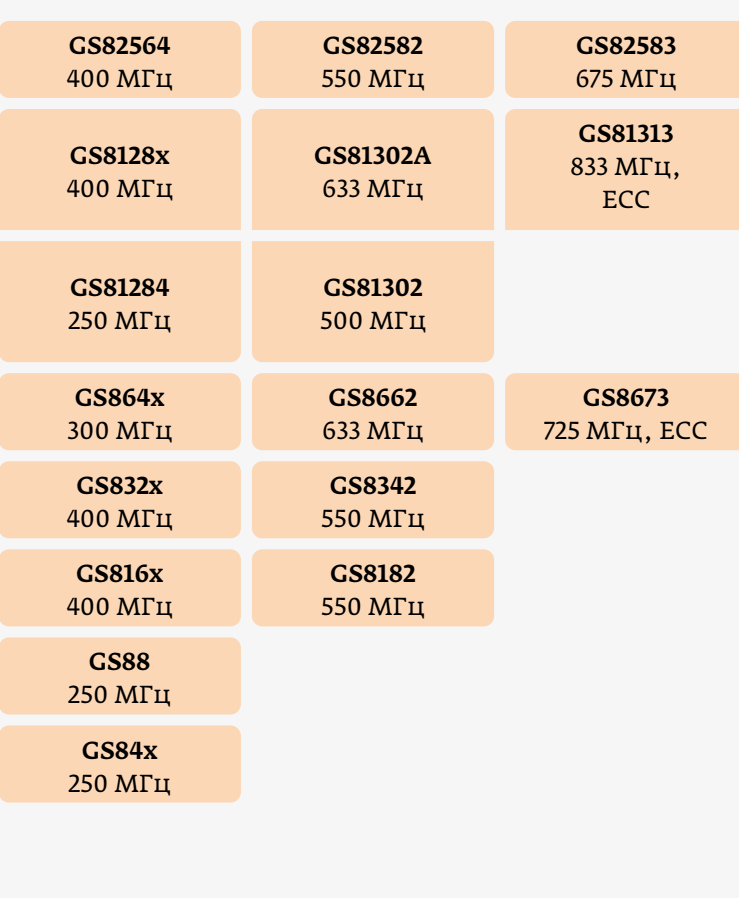

Рис. 2. Основные семейства микросхем GSI Technology

Таблица 1. Тип корпуса и объем памяти для разных семейств микросхем

\begin{tabular}{|c|c|c|c|c|}
\hline Тип корпуса & $\begin{array}{c}\text { NBT, } \\
\text { SyncBurst }\end{array}$ & $\begin{array}{c}\text { SigmaQuad-II/II+, } \\
\text { SigmaDDR-II/II+ }\end{array}$ & \multicolumn{2}{|c|}{$\begin{array}{c}\text { Габариты } \\
\text { корпуса }\end{array}$} \\
\hline \multirow{6}{*}{$\begin{array}{l}165-B G A \\
(15 \times 13 \mathrm{MM})\end{array}$} & 9 Мбит & - & \multirow{6}{*}{ 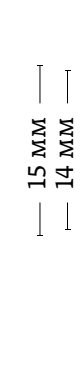 } & \multirow{6}{*}{ 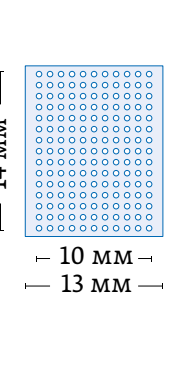 } \\
\hline & 18 Мбит & 18 Мбит & & \\
\hline & 36 Мбит & 36 Мбит & & \\
\hline & - & 72 Мбит & & \\
\hline & 144 Мбит & - & & \\
\hline & 288 Мбит & - & & \\
\hline \multirow{4}{*}{$\begin{array}{l}165-B G A \\
(17 \times 15 \mathrm{MM})\end{array}$} & 72 Мбит & - & \multirow{4}{*}{ 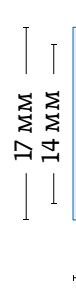 } & \\
\hline & - & 144 Мбит & & \\
\hline & \multirow[t]{2}{*}{ - } & 288 Мбит & & \\
\hline & & & & $\begin{array}{l}-10 \mathrm{MM}+ \\
-15 \mathrm{MM}-\end{array}$ \\
\hline
\end{tabular}

GSI Technology производит синхронную статическую память в ВGА-корпусах с содержанием свинца.

GSI Technology - единственный производитель сегодня, выпускающий SRAM третьего поколения SigmaQuad-III и SigmaDDR-III, а также микросхемы такого класса для температурных диапазонов $-40 \ldots 125^{\circ} \mathrm{C}$ и -55...125 C с гарантией последующего производства микросхем в течение 10 лет после размещения нового заказа.

Полезная опция, в частности для отечественных разработчиков, возможность заказа всех микросхем из одной партии и изготовления по индивидуальным требованиям заказчика. На сегодняшний день ни один производитель в мире не может предложить аналогичную 
Таблица 2. Сравнение производительности различных типов ОзУ

\begin{tabular}{|c|c|c|c|}
\hline Семейство & Макс. скорость доступа & Задержка чтения & макс. полоса пропускания \\
\hline $\begin{array}{l}\text { SigmaQuad/DDR } \\
\text { многобанковая }\end{array}$ & 2,66 млрд т/с @1333 МГц (non-random) & 5-6 нс & 192 гбит/с (х36) \\
\hline $\begin{array}{l}\text { SigmaQuad / DDR } \\
\text { однобанковая }\end{array}$ & 1,866 млрд т/с @ 933 МГц (random) & $5-6$ нс & 134 Гбит/с (х36) \\
\hline $\begin{array}{l}\text { NBT/SyncBurst } \\
\text { однобанковая }\end{array}$ & 400 млн т/с @ 400 МГц (random) & 5-6 нс & 14,4 Гбит / с (х36) \\
\hline $\begin{array}{l}\text { LLDRAM-II } \\
\text { многобанковая }\end{array}$ & $\begin{array}{l}533 \text { млн т/с @ } 533 \text { МГц (non-random) } \\
\text { 66,6 млн т/с @ } 533 \text { МГц (random) }\end{array}$ & $15 \mathrm{HC}$ & $\begin{array}{r}38,4 \text { Гбит / с (х36) } \\
\text { 4,8 Гбит/с (х36) }\end{array}$ \\
\hline $\begin{array}{l}\mathrm{DDR} 2 / 3 / 4 / 5 \\
\text { многобанковая }\end{array}$ & $\begin{array}{l}400 \text { млн т/с @1600 МГц (non-random) } \\
22 \text { млн т/с @1600 МГц (random) }\end{array}$ & $\begin{array}{l}13-15 \mathrm{HC} \\
40-45 \mathrm{HC}\end{array}$ & $\begin{array}{r}51,2 \text { Гбит/с (x16) } \\
2,8 \text { Гбит/с (x16) }\end{array}$ \\
\hline
\end{tabular}

гибкость. При этом ни одна из микросхем GSI Technology (в коммерческом, индустриальном исполнении или с расширенным температурным диапазоном) не подпадает под экспортные ограничения США или Европейского союза.

Кроме классической статической памяти, GSI также производит псевдоSRAM - Low Latency DRAM-II объемом 144 и 288 Мбит, время доступа которой не превышает 15 нс. В табл. 2 приведено сравнение характеристик различных типов памяти. Как видно из приведенных в таблице данных, задержка чтения синхронной статической SRAM не превышает 5-6 нс. Именно преимущество в скорости доступа и задержке чтения оправдывает высокую стоимость статической памяти в сравнении с DDR2/3/4/5. По соотношению цена / производительность семейство LLDRAM-II является компромиссным выбором между SRAM и DRAM.
С 2019 года компания начала выпуск радиационностойких микросхем NBT, SyncBurst и SigmaQuad-II+ в двух исполнениях: Rad-Hard и Rad-Tolerant. Функционально микросхемы двух исполнений являются полными аналогами. Однако для их производства используются различные кристаллы. Микросхемы Rad-Hard выпускаются в керамических корпусах, что обеспечивает более высокую надежность, и вместе с тем требуют получения экспортной лицензии для поставки в Российскую Федерацию. На микросхемы в исполнении Rad-Tolerant лицензии или другие документы, ограничивающие их применение, не распространяются. Основные характеристики радиационно-стойких микросхем GSI Technology приведены в табл. 3.

Микросхемы Rad-Hard и Rad-Tolerant изготавливаются по 40-нм технологии и успешно прошли сертификацию на соответствие требованиям QML-Q и QML-V по

Таблица 3. Радиационно-стойкие микросхемы

\begin{tabular}{|c|c|c|}
\hline Характеристика & Rad-Hard & Rad-Tolerant \\
\hline Объем, Мбит & \multicolumn{2}{|c|}{$\begin{array}{l}\text { 288 / } 144 \text { / } 72 \text { - SigmaQuad-II+ } \\
\text { 144 / } 72 \text { / } 36 \text { - NBT и SyncBurst }\end{array}$} \\
\hline Частота, МГц & \multicolumn{2}{|c|}{$\begin{array}{l}\text { 350/250 - SigmaQuad-II+ } \\
\text { 333/250 - NBT и SyncBurst }\end{array}$} \\
\hline Разрядность & \multicolumn{2}{|c|}{$\mathrm{x} 18 / \mathrm{x} 36$} \\
\hline Корпус & $\begin{array}{c}\text { 165-CCGA - SigmaQuad-II+ } \\
\text { 165-LCA - SigmaQuad-II+ } \\
\text { 100-Ceramic QFP - SyncBurst и NBT }\end{array}$ & $\begin{array}{l}\text { 165-LBGA - SigmaQuad-II+ } \\
\text { 100-TQFP - SyncBurst и NBT }\end{array}$ \\
\hline Общая накопленная доза & $>300$ кРад & $>50$ кРад \\
\hline Защита от тиристорного эффекта & $80 \mathrm{MэB} \cdot \mathrm{CM}^{2} / \mathrm{M \Gamma}\left(125^{\circ} \mathrm{C}\right)$ & $42,2 \mathrm{M} \ni \mathrm{B} \cdot \mathrm{CM}^{2} / \mathrm{M \Gamma}\left(125^{\circ} \mathrm{C}\right)$ \\
\hline Экспортный код (ECCN) & 9А515 - лицензия & 3А991.a.2.b-без лицензии \\
\hline
\end{tabular}


процедурам, изложенным в спецификации MIL-PRF-38535. Техническую документацию на микросхемы можно загрузить с сайта производителя.

Выход на рынок радиационно-стойких микросхем GSI стал настоящим прорывом в отрасли, поскольку по объему памяти и производительности новые микросхемы многократно превосходят имеющиеся на рынке предложения от других производителей. Основные применения радиационно-стойких микросхем GSI: датчики звездного неба, приемо-передающие устройства телеметрии, вычислители для дистанционного зондирования земли, а также датчико-преобразующая аппаратура для ракетно-космической техники.

\section{ІР-КОНТРОЛЛЕР ДЛЯ ПОДКЛЮЧЕНИЯ SRAM К ПЛИС}

GSI Technology - единственный производитель SRAM, который разрабатывает и распространяет IР-контроллеры для подключения микросхем памяти к Плис.

Следует отметить, что асинхронная память не требует разработки ІР-контроллера. Как правило, он уже аппаратно реализован в процессоре. Однако по-другому обстоят дела с синхронной памятью. Практически в каждом устройстве SRAM применяется в качестве внешней памяти для ПЛИС и управляется с помощью ІР-контроллера.

IP-контроллер для средне-производительной памяти NBT и SyncBurst адаптировать под свою систему относительно несложно - его можно скачать с веб-страницы каждой микросхемы. IР-контроллеры для семейств SigmaQuad и SigmaDDR требуют более тонкой настройки. Поскольку GSI производит микросхемы с наивысшими техническими показателями, компания хочет быть уверенной, что ее клиенты получают максимум от микросхем. По этой причине GSI предоставляет контроллеры, уже сконфигурированные под конкретную задачу клиента. Средний срок подготовки контроллера составляет около двух недель. Контроллер передается в исходном коде на языке программирования Плис Verilog или VHDL, а также сопровождается инструкцией по его настройке.

В настоящее время разработаны ІР-контроллеры для плИС производства Xilinx, а именно Virtex, Kintex и Zynq серий 6 и 7, Ultrascale и Ultrascale+, а также для радиационно-стойких Плис Xilinx Virtex 5/ SIRF и Microsemi RTG4.

Все контроллеры проверены на отладочных платах собственного производства (рис. 3). Компания предлагает примеры проектов на базе микросхем GSI, включая электрическую схему, топологию и перечень элементов отладочной платы. Также на веб-странице микросхемы доступны для скачивания модели ModelSim, Test Bench и IBIS. По запросу предоставляются отчеты о надежности FIT или MTTF.

\section{ДЕТЕКТОР ДЛЯ МАШИННОГО ЗРЕНИЯ}

В настоящее время компания сконцентрировала свои усилия на развитии нового продукта - нейросетевого детектора для искусственного интеллекта Associative Processing Unit (APU) (рис. 4)

В системах машинного зрения возрастают требования к количеству и скорости одновременно распознаваемых объектов. Несмотря на то, что для реализации большинства приложений достаточно производительности микроконтроллера, требования к системе критически возрастают, когда необходимо идентифицировать несколько десятков, сотен, а порой и тысячи объектов в режиме реального времени. При этом размер базы данных может достигать нескольких миллиардов характеристических векторов (в англоязычной литературе-embeddings). В качестве примера можно упомянуть задачи распознавания людей в системе "умного" города и поиск активного вещества в хемоинформатике

Традиционные решения для поиска схожих векторов из базы с вектором нового объекта (в англоязычной литературе такая задача называется similarity search), peaлизованы на основе ЦП. Однако, из-за ограничений,

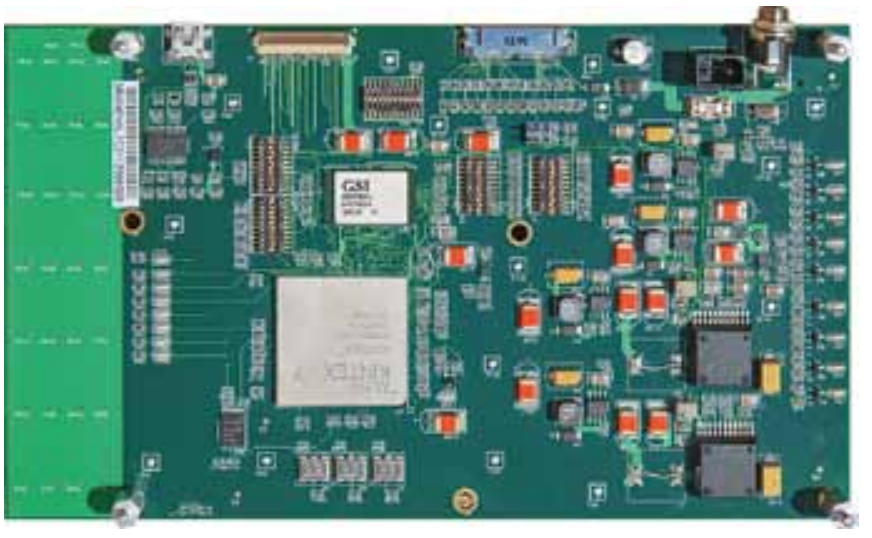

Рис. 3. Отладочная плата для SigmaQuad-II+

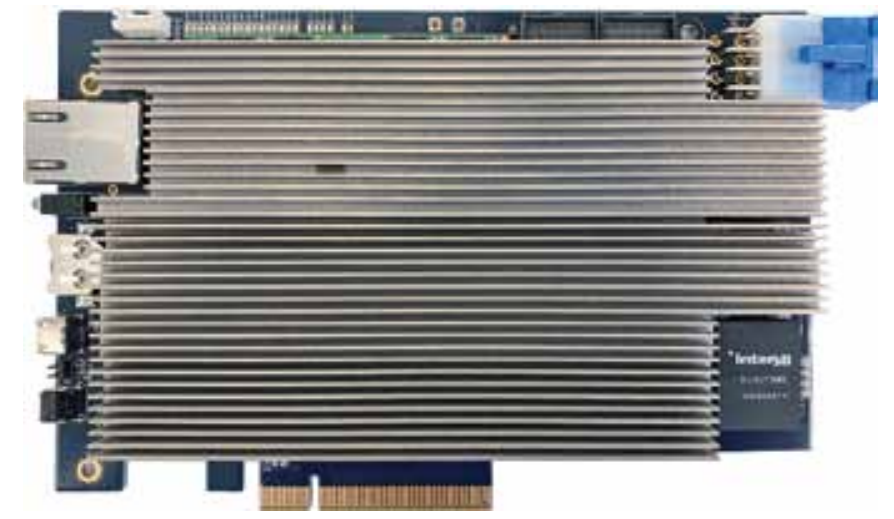

Рис. 4. Нейросетевой детектор от CSI Technology 
присущих аппаратной архитектуре цП, операции сравнения выполняются последовательно в каждом ядре. Ввиду ограниченного количества ядер производительность всей системы существенно снижается. Для достижения необходимой скорости разработчики вынуждены закупать новые серверы и масштабировать систему, что в конечном итоге приводит к значительному удорожанию проекта и периодическим выходам из строя отдельных модулей.

В отличие от ЦП, детектор от GSI Technology обрабатывает до 10 тыс. одновременных запросов, а также обеспечивает поиск по нескольким базам одновременно, благодаря чему производительность APU в несколько десятков раз выше, чем производительность современного ЦП. При этом потребляемая мощность APU в активном режиме в 3,5 раза ниже, что позволяет сэкономить не только на системе кондиционирования сервера, но также на системе электропитания. Таким образом, вместо того, чтобы нагружать основной процессор поиском похожих векторов, пользователь может делегировать эту задачу в APU и получить результат гораздо быстрее, сэкономив бюджет проекта и затраты на его последующее обслуживание. Выходное сообщение APU содержит индексы наиболее схожих векторов из базы и расстояния до них. Для хранения характеристических векторов базы данных в APU предусмотрена перезаписываемая память объемом 16 Гбайт. В детекторе реализована поддержка векторов в формате FP32×64/128/256/512/1024 и бинарной форме $(64,128,256,512,1024,2048,4096)$.

Компания предполагает, что детекторы позволят значительно увеличить производительность систем не только в инфраструктуре «умного»города, но и в таких областях, как электронная коммерция (е-commerce), розничная торговля (анализ продуктовых остатков в магазинах), биотехнология (сравнения молекулярных структур при разработке вакцин), а также обработка естественного языка. Дополнительную информацию о детекторе можно получить на сайте GSI Technology.

$$
\because *
$$

Официальными дистрибьюторами GSI Technology в Pocсийской Федерации являются компании Avnet Silica и Mouser Electronics в лице авторизованного поставщика «ПМ Электроникс».

В следующих статьях о продукции компании GSI Technology будут подробно рассмотрены основные семейства SRAM, их различия и особенности в сравнении с аналогичными микросхемами других производителей. Отдельная статья будет посвящена вычислительному модулю для машинного зрения.

\section{КНИГИ ИЗДАТЕЛЬСТВА "ТЕХНОСФЕРА»}

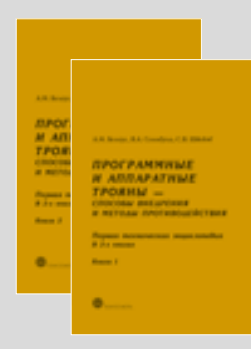

Цена за две книги 2400 руб.

\section{ПРОГРАММНЫЕ И АППАРАТНЫЕ ТРОЯНЫ - СПОСОБЫ ВНЕДРЕНИЯ И МЕТОДЫ ПРОТИВОДЕЙСТВИЯ. ПЕРВАЯ ТЕХНИЧЕСКАЯ ЭНЦИКЛОПЕДИЯ}

В 2-х книгах
Белоус А. И., Солодуха В. А., Шведов С. В. Под общей редакцией Белоуса А. И.

\begin{abstract}
В двухтомнике исследован феномен программных и аппаратных троянов, которые фактически являются технологической платформой современного и перспективного кибероружия. В первой вводной главе показано, что развитие всех “обычных» и «новейших» видов вооружений дошло до такой стадии, что их использование на практике будет равносильно самоубийству начавшей войну стороны. Осознание этого факта привело к развитию информационно-технического оружия (кибероружия и нейрооружия). В последующих главах детально исследованы концепции, методы и примеры реализации этого вида оружия. Рассмотрены основные виды программных троянов, вирусов и шпионских программ, показан эволюционный путь развития аппаратных троянов от “ящиков" и “коробочек» до микросхем.

Книга ориентирована на специалистов по информационной безопасности, а также будет полезна всем интересующимся данной темой.
\end{abstract}

